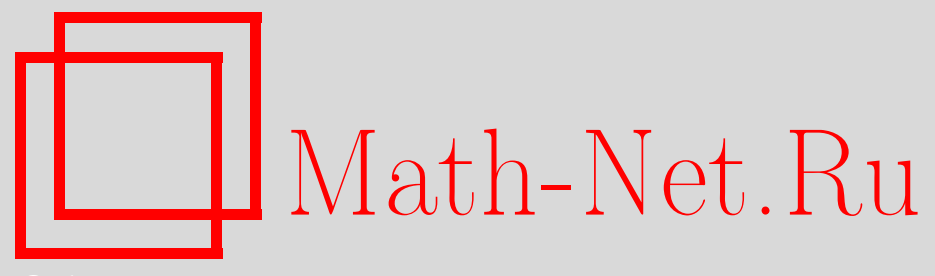

В. О. Лукащук, С. Ю. Лукащук, Групповая классификация, инвариантные решения и законы сохранения нелинейного двумерного ортотропного уравнения фильтрации с дробной производной Римана-Лиувилля по времени, Вестн. Сам. гос. техн. ун-та. Сер. Физ.-мат. науки, 2020, номер 2, 226-248

DOI: https://doi.org/10.14498/vsgtu1761

Использование Общероссийского математического портала MathNet.Ru подразумевает, что вы прочитали и согласны с пользовательским соглашением

http://www.mathnet.ru/rus/agreement

Параметры загрузки:

IP: 35.173 .137 .237

26 апреля 2023 г., 15:33:27

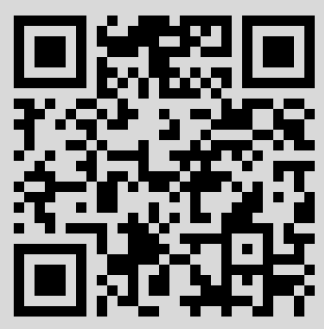




\title{
Групповая классификация, инвариантные решения и законы сохранения нелинейного двумерного ортотропного уравнения фильтрации с дробной производной Римана-Лиувилля по времени
}

\author{
В. О. Лукащук, С. Ю. Лукащук
}

Уфимский государственный авиационный технический университет, Россия, 450008, Уфа, ул. К. Маркса, 12.

\section{Аннотация}

Рассматривается нелинейное двумерное ортотропное уравнение фильтрации с дробной производной Римана-Лиувилля по времени. Доказывается, что такое уравнение может допускать группы точечных преобразований только линейно-автономного типа. Решается задача групповой классификации рассматриваемого уравнения по его точечным симметриям относительно коэффициентов пьезопроводности, являющихся функциями квадрата модуля градиента давления. Доказывается, что если порядок дробного дифференцирования меньше единицы, основная допускаемая уравнением группа точечных преобразований является четырехпараметрической и расширяется до пятипараметрической в изотропном случае. Для степенных зависимостей коэффициентов пьезопроводности допускаемая группа становится пятипараметрической в ортотропном случае и шестипараметрической в изотропном случае. Также выделяется специальный вид степенной зависимости коэффициентов, не имеющий аналога в случае классического уравнения фильтрации, при котором происходит дополнительное расширение группы оператором проективного преобразования. Для уравнения с порядком дробного дифференцирования $\alpha \in(1,2)$ размерности всех допускаемых групп оказываются на единицу больше за счет допускаемого оператора, соответствующего сдвигу решения на дополнительное частное решение этого уравнения.

На основе проведенной групповой классификации для соответствующих алгебр Ли инфинитезимальных операторов групп точечных преобразований, допускаемых различными видами рассматриваемого нели-

\section{Научная статья}

(웅 Контент публикуется на условиях лицензии Creative Commons Attribution 4.0 International (https://creativecommons.org/licenses/by/4.0/deed.ru)

\section{Образец для цитирования}

Лукащук В. О., Лукащук С. Ю. Групповая классификация, инвариантные решения и законы сохранения нелинейного двумерного ортотропного уравнения фильтрации с дробной производной Римана-Лиувилля по времени // Вестн. Сам. гос. техн. ун-та. Сер. Физ.-мат. науки, 2020. Т. 24, № 2. С. 226-248. doi: 10.14498/vsgtu1761.

\section{Сведения об авторах}

Вероника Олеговна Лукащук (D) https://orcid.org/0000-0002-3082-1446

кандидат физико-математических наук; доцент; каф. высокопроизводительных вычислительных технологий и систем; e-mail: voluks@gmail.com

Станислав Юръевич Лукащук (1) https://orcid.org/0000-0001-9209-5155

доктор физико-математических наук; профессор; каф. высокопроизводительных вычислительных технологий и систем; e-mail: Isu@ugatu.su 
нейного ортотропного дробно-дифференциального уравнения, выписываются представления инвариантно-групповых решений, соответствующие оптимальным системам двумерных подалгебр. Приводятся примеры уравнений, получающихся в результате симметрийной редукции исходного дробно-дифференциального уравнения, а также некоторые их решения.

Доказывается, что рассматриваемое дробно-дифференциальное уравнение фильтрации является нелинейно самосопряженным, что дает возможность строить его законы сохранения. Соответствующие компоненты всех найденных сохраняющихся векторов приводятся в явном виде.

Ключевые слова: дробно-дифференциальное уравнение фильтрации, групповая классификация, точечная симметрия, инвариантное решение, закон сохранения.

Получение: 29 ноября 2019 г. / Исправление: 17 мая 2020 г.

Принятие: 1 июня 2020 г. / Публикация онлайн: 30 июня 2020 г.

Введение. Интегро-дифференциальные уравнения с производными дробных порядков различных типов $[1,2]$ в последнее время привлекают большое внимание исследователей из самых различных областей науки и техники благодаря возможности их использования в качестве математических моделей сложных процессов, сред и систем, проявляющих эффекты памяти и пространственной нелокальности [3-12]. Наиболее хорошо в настоящее время исследованы одномерные дробно-дифференциальные уравнения диффузионного типа, к которому относится и большинство простейших дробно-дифференциальных уравнений фильтрации. Однако с точки зрения практического использования существенно больший интерес представляют уравнения в двумерных и трехмерных областях. Исследование ряда важных качественных свойств таких уравнений, особенно нелинейных, может быть выполнено методами современного группового анализа [13-17]. Для дробно-дифференциальных уравнений в последнее десятилетие удалось развить ряд классических теоретико-групповых методов (см. $[18,19]$ и цитируемую там литературу), что дало возможность находить допускаемые такими уравнениями группы симметрий, решать задачи их групповой классификации, строить факторуравнения, инвариантные решения и законы сохранения. Отметим, что задача групповой классификации уравнения имеет не только теоретическое, но и прикладное значение, поскольку выделение классов уравнений с расширенной группой симметрий позволяет выделять также и соответствующие подмодели, обладающие расширенным набором симметрийных свойств и, как следствие, имеющих дополнительные инвариантные решения и законы сохранения.

Настоящая работа посвящена исследованию симметрийных свойств нелинейного двумерного дробно-дифференциального уравнения

$$
{ }_{0} D_{t}^{\alpha} u=\left(f\left(u_{x}^{2}+u_{y}^{2}\right) u_{x}\right)_{x}+\left(g\left(u_{x}^{2}+u_{y}^{2}\right) u_{y}\right)_{y}, \quad f, g>0, \quad \alpha \in(0,1) \cup(1,2) .
$$

Здесь через ${ }_{0} D_{t}^{\alpha}$ обозначен оператор дробного дифференцирования Римана- 
Лиувилля порядка $\alpha$ :

$$
{ }_{0} D_{t}^{\alpha} u=\frac{1}{\Gamma(n-\alpha)} \frac{\partial^{n}}{\partial t^{n}} \int_{0}^{t} \frac{u(\tau, x, y)}{(t-\tau)^{\alpha-n+1}} d \tau, \quad n=[\alpha]+1 .
$$

Уравнение (1) позволяет, в частности, описывать процессы фильтрации в ортотропной гетерогенной пористой среде и может быть получено на основе дробно-дифференциальных модификаций закона Дарси [20-22] (в этом случае в качестве функции $u$ выступает давление, а функции $f$ и $g$ являются компонентами тензора пьезопроводности ортотропной среды). При $\alpha=1$ уравнение (1) превращается в классическое нелинейное двумерное ортотропное уравнение фильтрации, результаты групповой классификации которого приведены в [23].

Решается задача групповой классификации уравнения (1) относительно функций $f$ и $g$ по допускаемым этим уравнением однопараметрическим группам Ли точечных преобразований вида

$$
\begin{array}{ll}
\bar{t}=\lambda(t, x, y, u, a), & \bar{y}=\nu^{2}(t, x, y, u, a), \\
\bar{x}=\nu^{1}(t, x, y, u, a), & \bar{u}=\omega(t, x, y, u, a), \\
\left.\lambda\right|_{a=0}=t,\left.\quad \nu^{1}\right|_{a=0}=x, & \left.\nu^{2}\right|_{a=0}=y,\left.\quad \omega\right|_{a=0}=u,
\end{array}
$$

где $a$ - параметр группы. Порядок дробного дифференцирования $\alpha$ не преобразуется. Группе преобразований (2) соответствует инфинитезимальный опеpaтор

$$
X=\tau(t, x, y, u) \frac{\partial}{\partial t}+\xi^{1}(t, x, y, u) \frac{\partial}{\partial x}+\xi^{2}(t, x, y, u) \frac{\partial}{\partial y}+\eta(t, x, y, u) \frac{\partial}{\partial u}
$$

координаты которого определяются соотношениями

$$
\begin{aligned}
& \tau(t, x, y, u)=\left.\frac{\partial \lambda(t, x, y, u, a)}{\partial a}\right|_{a=0}, \quad \xi^{2}(t, x, y, u)=\left.\frac{\partial \nu^{2}(t, x, y, u, a)}{\partial a}\right|_{a=0}, \\
& \xi^{1}(t, x, y, u)=\left.\frac{\partial \nu^{1}(t, x, y, u, a)}{\partial a}\right|_{a=0}, \quad \eta(t, x, y, u)=\left.\frac{\partial \omega(t, x, y, u, a)}{\partial a}\right|_{a=0} .
\end{aligned}
$$

Такой инфинитезимальный оператор допускаемой уравнением группы будет, как обычно, называться точечной симметрией этого уравнения.

1. О линейной автономности группы, допускаемой дробно-дифференциальным уравнением. Уравнение (1) является частным случаем уравнения вида

$$
{ }_{0} D_{t}^{\alpha} u=\Phi\left(t, x, y, u, u_{x}, u_{y}, u_{x x}, u_{x y}, u_{y y}\right)
$$

для симметрий которого может быть доказано одно важное свойство.

Следуя работе [24], преобразования вида (2), в которых функции $\lambda, \nu^{1}$ и $\nu^{2}$ не зависят от $u$, будем называть $x$-автономными преобразованиями. Очевидно, что в этом случае координаты $\tau, \xi^{1}$ и $\xi^{2}$ оператора (3) также не будут зависеть от $u$. Соответствующие симметрии будут называться $x$-aвmoномными симметриями. Если для $x$-автономной симметрии дополнительно 
выполнено условие $\eta_{u и}=0$, то такая симметрия будет называться линейноавтономной [25].

Теорема 1. Уравнение (4) может обладать точечными симметриями вида (3) только линейно-автономного типа с координатами

$$
\begin{gathered}
\tau=\rho(x, y) t+\phi(x, y) t^{2}, \quad \xi^{1}=\theta^{1}(x, y), \quad \xi^{2}=\theta^{2}(x, y) \\
\eta=\psi(t, x, y)+[\varphi(x, y)+(\alpha-1) \phi(x, y) t] u
\end{gathered}
$$

где $\rho, \phi, \theta^{1}, \theta^{2}, \psi, \varphi-$ произволъные функиии своих аргументов.

Доказательств о. Необходимое условие инвариантности [19] уравнения (4) относительно группы точечных преобразований, определяемой оператором (3), может быть записано в виде

$$
\left[X_{(\alpha) 0} D_{t}^{\alpha} u-X_{(2)} \Phi\right]_{(4)}=0
$$

где

$$
X_{(\alpha)}=X+\zeta_{(\alpha)} \frac{\partial}{\partial D_{t}^{\alpha} u}
$$

- продолжение оператора (3) на дробно-дифференциальную переменную $D_{t}^{\alpha} u$, а $X_{(2)}$ - классическое второе продолжение оператора (3) на все аргументы функции $\Phi$ (см., например, $[13,17])$. Координата $\zeta_{(\alpha)}$ находится по формуле продолжения [19]:

$$
\zeta_{(\alpha)}=D_{t}^{\alpha}(W)+\tau D_{t}^{\alpha+1} u+\xi^{1} D_{t}^{\alpha} u_{x}+\xi^{2} D_{t}^{\alpha} u_{y}
$$

где $W=\eta-\tau u_{t}-\xi^{1} u_{x}-\xi^{2} u_{y}$.

Как доказано в [26] (см. также [18,19]), определяющее уравнение (6) должно быть дополнено условием неподвижности точки начала отсчета дробной производной под действием преобразований группы:

$$
\left.\tau(t, x, y, u)\right|_{t=0}=0 .
$$

Функция $\eta$ является сложной функцией переменной $t$, поэтому вычисление дробной производной $D_{t}^{\alpha} \eta$, входящей в $(7)$, является непростой задачей. Как показано в [18], такая производная будет представляться рядом с четырехкратным вложенным суммированием. Поэтому для упрощения доказательства теоремы воспользуемся следующим формальным приемом. Перепишем функцию $\eta$ в квазилинейном по переменной $u$ виде

$$
\eta(t, x, y, u)=\psi(t, x, y)+\tilde{\eta}(t, x, y, u) u
$$

Тогда для доказательства утверждения теоремы необходимо показать, что $\tilde{\eta}_{u}=0$.

Рассмотрим первое слагаемое в определяющем уравнении (6) с учетом формулы продолжения (7):

$$
\begin{aligned}
X_{(\alpha) 0} D_{t}^{\alpha} u=\zeta_{(\alpha)} \equiv{ }_{0} D_{t}^{\alpha}(\psi+\tilde{\eta} u & \left.-\tau u_{t}-\xi^{1} u_{x}-\xi^{2} u_{y}\right)+ \\
& +\tau_{0} D_{t}^{\alpha+1}(u)+\xi^{1}{ }_{0} D_{t}^{\alpha}\left(u_{x}\right)+\xi^{2}{ }_{0} D_{t}^{\alpha}\left(u_{y}\right) .
\end{aligned}
$$


Как это принято в групповом анализе, будем полагать, что все координаты инфинитезимального оператора (3) являются требуемое число раз непрерывно дифференцируемыми по своим аргументам. Тогда возможно применение оператора дробного дифференцирования ${ }_{0} D_{t}^{\alpha}$ независимо к каждому из слагаемых, стоящих в круглых скобках в правой части (9), а также использование обобщенного правила Лейбница [1]:

$$
{ }_{0} D_{t}^{\alpha}(F G)=\sum_{k=0}^{\infty}\left(\begin{array}{l}
\alpha \\
k
\end{array}\right){ }_{0} D_{t}^{\alpha-k}(F) D_{t}^{k}(G),
$$

где ${ }_{0} D_{t}^{\alpha-k}(F)$ - дробные производные (при $\alpha-k>0$ ) и дробные интегралы (при $\alpha-k<0)$ соответствующих порядков, $\left(\begin{array}{l}\alpha \\ k\end{array}\right)=\frac{\Gamma(\alpha+1)}{k ! \Gamma(\alpha-k+1)}$ - биномиальные коэффициенты. Также воспользуемся очевидным равенством

$$
\tau u_{t}=D_{t}(\tau u)-D_{t}(\tau) u \text {. }
$$

В силу условия (8) и регулярности функции $\tau$ и ее первой производной по $t$ в точке $t=0$ имеем $\left.\tau u\right|_{t=0}=0$. Тогда (см. [1,2]),

$$
{ }_{0} D_{t}^{\alpha} D_{t}(\tau u)={ }_{0} D_{t}^{\alpha+1}(\tau u) .
$$

Подстановка приведенных выражений в (9) и применение (10) дает

$$
\begin{aligned}
\zeta_{(\alpha)}={ }_{0} D_{t}^{\alpha}(\psi) & +\left[\tilde{\eta}-\alpha D_{t}(\tau)\right]_{0} D_{t}^{\alpha}(u)+ \\
& +\sum_{k=1}^{\infty}\left(\begin{array}{l}
\alpha \\
k
\end{array}\right){ }_{0} D_{t}^{\alpha-k}(u) D_{t}^{k}(\tilde{\eta})-\sum_{k=1}^{\infty}\left(\begin{array}{c}
\alpha \\
k+1
\end{array}\right){ }_{0} D_{t}^{\alpha-k}(u) D_{t}^{k+1}(\tau)- \\
& -\sum_{k=1}^{\infty}\left(\begin{array}{l}
\alpha \\
k
\end{array}\right){ }_{0} D_{t}^{\alpha-k}\left(u_{x}\right) D_{t}^{k}\left(\xi^{1}\right)-\sum_{k=1}^{\infty}\left(\begin{array}{l}
\alpha \\
k
\end{array}\right){ }_{0} D_{t}^{\alpha-k}\left(u_{y}\right) D_{t}^{k}\left(\xi^{2}\right) .
\end{aligned}
$$

Подставляя $(11)$ в (6) и заменяя дробную производную ${ }_{0} D_{t}^{\alpha}(u)$ на $\Phi$ в силу исходного уравнения (4), приходим к следующему определяющему уравнению:

$$
\begin{aligned}
{ }_{0} D_{t}^{\alpha}(\psi) & +\left[\tilde{\eta}-\alpha D_{t}(\tau)\right] \Phi+ \\
& +\sum_{k=1}^{\infty}\left(\begin{array}{l}
\alpha \\
k
\end{array}\right){ }_{0} D_{t}^{\alpha-k}(u) D_{t}^{k}(\tilde{\eta})-\sum_{k=1}^{\infty}\left(\begin{array}{c}
\alpha \\
k+1
\end{array}\right){ }_{0} D_{t}^{\alpha-k}(u) D_{t}^{k+1}(\tau)- \\
& -\sum_{k=1}^{\infty}\left(\begin{array}{l}
\alpha \\
k
\end{array}\right){ }_{0} D_{t}^{\alpha-k}\left(u_{x}\right) D_{t}^{k}\left(\xi^{1}\right)-\sum_{k=1}^{\infty}\left(\begin{array}{l}
\alpha \\
k
\end{array}\right){ }_{0} D_{t}^{\alpha-k}\left(u_{y}\right) D_{t}^{k}\left(\xi^{2}\right)=X_{(2)} \Phi .
\end{aligned}
$$

Выполним расщепление уравнения (12) по всем интегралам и производным дробных порядков, которые могут рассматриваться теперь в качестве независимых переменных.

Расщепление по ${ }_{0} D_{t}^{\alpha-k}\left(u_{x}\right)$ и ${ }_{0} D_{t}^{\alpha-k}\left(u_{y}\right)$ приводит к уравнениям

$$
D_{t}^{k}\left(\xi^{1}\right)=0, \quad D_{t}^{k}\left(\xi^{2}\right)=0, \quad k=1,2, \ldots,
$$


интегрирование которых дает

$$
\xi^{1}=\theta^{1}(x, y), \quad \xi^{2}=\theta^{2}(x, y)
$$

где $\theta^{1}$ и $\theta^{2}$ - произвольные функции.

Аналогично, расщепление $(12)$ по ${ }_{0} D_{t}^{\alpha-k}(u)$ приводит к системе

$$
(k+1) D_{t}^{k}(\tilde{\eta})-(\alpha-k) D_{t}^{k+1}(\tau)=0, \quad k=1,2, \ldots
$$

Рассматривая первые два уравнения этой системы (соответствующие $k=1$ и $k=2$ ), находим

$$
D_{t}^{2}(\tilde{\eta})=0
$$

или

$$
\tilde{\eta}_{t t}+2 \tilde{\eta}_{t u} u_{t}+\tilde{\eta}_{u u} u_{t}^{2}+\tilde{\eta}_{u} u_{t t}=0
$$

Расщепление этого уравнения по $u_{t}$ и $u_{t t}$ дает требуемое условие $\tilde{\eta}_{u}=0$. Решение оставшегося уравнения $\tilde{\eta}_{t t}=0$ может быть представлено в виде

$$
\tilde{\eta}=\vartheta(x, y) t+\varphi(x, y)
$$

где $\vartheta(x, y)$ и $\varphi(x, y)$ - произвольные функции. Тогда уравнение, соответствующее $k=1$, принимает вид

$$
(\alpha-1) D_{t}^{2} \tau=2 \vartheta(x, y) .
$$

Его интегрирование с учетом условия (8) дает

$$
\tau=\phi(x, y) t^{2}+\rho(x, y) t
$$

где $\rho(x, y)$ - произвольная функция, а $\phi(x, y)=\vartheta(x, y) /(\alpha-1)$.

В результате приходим к искомым представлениям (5). Определяющее уравнение (12) принимает при этом вид

$$
{ }_{0} D_{t}^{\alpha}(\psi)+[\varphi-\alpha \rho-(1+\alpha) \phi t] \Phi=X_{(2)} \Phi .
$$

Результат теоремы 3 можно усилить, если наложить дополнительные ограничения на вид функции $\Phi$. Заметим, что функция $\Phi$ не зависит от $u_{t}$ и ее производных. Следовательно, правая часть уравнения (13) будет линейно зависеть от переменных $u_{t}, u_{t x}$ и $u_{t y}$ в силу линейной зависимости от них координат продолженного оператора $X_{(2)}$. Осуществляя дополнительное расщепление уравнения (13) по этим переменным, приходим к следующей системе уравнений:

$$
\begin{aligned}
& \tau_{x} \frac{\partial \Phi}{\partial u_{x}}+\tau_{y} \frac{\partial \Phi}{\partial u_{y}}+\tau_{x x} \frac{\partial \Phi}{\partial u_{x x}}+\tau_{x y} \frac{\partial \Phi}{\partial u_{x y}}+\tau_{y y} \frac{\partial \Phi}{\partial u_{y y}}=0 \\
& 2 \tau_{x} \frac{\partial \Phi}{\partial u_{x x}}+\tau_{y} \frac{\partial \Phi}{\partial u_{x y}}=0 \\
& \tau_{x} \frac{\partial \Phi}{\partial u_{x y}}+2 \tau_{y} \frac{\partial \Phi}{\partial u_{y y}}=0
\end{aligned}
$$


Из двух последних уравнений этой системы следует, что при выполнении условия

$$
4 \frac{\partial \Phi}{\partial u_{x x}} \frac{\partial \Phi}{\partial u_{y y}}-\left(\frac{\partial \Phi}{\partial u_{x y}}\right)^{2} \neq 0
$$

справедливы равенства $\tau_{x}=\tau_{y}=0$. Тогда в (5) имеем $\rho=C_{1}, \phi=C_{2}$, и

$$
\begin{gathered}
\tau=C_{1} t+C_{2} t^{2}, \quad \xi^{1}=\theta^{1}(x, y), \quad \xi^{2}=\theta^{2}(x, y), \\
\eta=\psi(t, x, y)+\left(\varphi(x, y)+(\alpha-1) C_{2} t\right) u,
\end{gathered}
$$

где $C_{1}$ и $C_{2}$ - произвольные постоянные.

2. Групповая классификация уравнения (1). Уравнение (1) может быть представлено в виде

$$
{ }_{0} D_{t}^{\alpha} u=F\left(u_{x}, u_{y}\right) u_{x x}+G\left(u_{x}, u_{y}\right) u_{y y}+H\left(u_{x}, u_{y}\right) u_{x y},
$$

где

$$
F=2 u_{x}^{2} f^{\prime}+f, \quad G=2 u_{y}^{2} g^{\prime}+g, \quad H=2 u_{x} u_{y}\left(f^{\prime}+g^{\prime}\right) .
$$

Для уравнения (17) условие (15) принимает вид $4 F G \neq H^{2}$. С учетом (18) нетрудно показать, что это условие нарушается только в том случае, когда $f(r)=g(r)=f_{0} / \sqrt{r}, f_{0}$ - произвольная постоянная. Однако подстановка этих функций в первое уравнение системы (14) снова приводит к уравнениям $\tau_{x}=\tau_{y}=0$. Таким образом, представления (16) справедливы для уравнения (1) при любых $f, g>0$.

Для уравнения (17) с учетом (16) уравнение (13) для определения функций $\theta^{1}, \theta^{2}, \varphi$ и $\psi$ принимает вид

$$
\begin{aligned}
{ }_{0} D_{t}^{\alpha}(\psi)+\left[\varphi-\alpha C_{1}-(1+\alpha) t C_{2}\right] & \left(F u_{x x}+G u_{y y}+H u_{x y}\right)- \\
-\zeta_{11} F-\zeta_{22} G-\zeta_{12} H & -\zeta_{1}\left(\frac{\partial F}{\partial u_{x}} u_{x x}+\frac{\partial G}{\partial u_{x}} u_{y y}+\frac{\partial H}{\partial u_{x}} u_{x y}\right)- \\
& -\zeta_{2}\left(\frac{\partial F}{\partial u_{y}} u_{x x}+\frac{\partial G}{\partial u_{y}} u_{y y}+\frac{\partial H}{\partial u_{y}} u_{x y}\right)=0
\end{aligned}
$$

в котором

$$
\begin{aligned}
& \zeta_{1}=\psi_{x}+\varphi_{x} u+\left(\varphi+(\alpha-1) C_{2} t-\theta_{x}^{1}\right) u_{x}-\theta_{x}^{2} u_{y}, \\
& \zeta_{2}=\psi_{y}+\varphi_{y} u-\theta_{y}^{1} u_{x}+\left(\varphi+(\alpha-1) C_{2} t-\theta_{y}^{2}\right) u_{y}, \\
& \begin{array}{c}
\zeta_{11}=\psi_{x x}+\varphi_{x x} u+\left(2 \varphi_{x}-\theta_{x x}^{1}\right) u_{x}-\theta_{x x}^{2} u_{y}+ \\
+\left(\varphi+(\alpha-1) C_{2} t-2 \theta_{x}^{1}\right) u_{x x}-2 \theta_{x}^{2} u_{x y}, \\
\zeta_{12}=\psi_{x y}+\varphi_{x y} u+\left(\varphi_{y}-\theta_{x y}^{1}\right) u_{x}+\left(\varphi_{x}-\theta_{x y}^{2}\right) u_{y}- \\
-\theta_{y}^{1} u_{x x}+\left(\varphi+(\alpha-1) C_{2} t-\theta_{x}^{1}+\theta_{y}^{2}\right) u_{x y}-\theta_{x}^{2} u_{y y}, \\
\zeta_{22}=\psi_{y y}+\varphi_{y y} u-\theta_{y y}^{1} u_{x}+\left(2 \varphi_{y}-\theta_{y y}^{2}\right) u_{y}- \\
-2 \theta_{y}^{1} u_{x y}+\left(\varphi+(\alpha-1) C_{2} t-2 \theta_{y}^{2}\right) u_{y y} .
\end{array}
\end{aligned}
$$


Расщепление (19) по $u, u u_{x x}, u u_{y y}$ и $u u_{z z}$ приводит к системе

$$
\begin{aligned}
& \varphi_{x} \frac{\partial F}{\partial u_{x}}+\varphi_{y} \frac{\partial F}{\partial u_{y}}=0, \\
& \varphi_{x} \frac{\partial G}{\partial u_{x}}+\varphi_{y} \frac{\partial G}{\partial u_{y}}=0, \\
& \varphi_{x} \frac{\partial H}{\partial u_{x}}+\varphi_{y} \frac{\partial H}{\partial u_{y}}=0, \\
& \varphi_{x x} F+\varphi_{y y} G+\varphi_{x y} H=0,
\end{aligned}
$$

расщепление по $t u_{x x}, t u_{y y}, t u_{z z}$ дает

$$
\begin{aligned}
& C_{2}\left(u_{x} \frac{\partial F}{\partial u_{x}}+u_{y} \frac{\partial F}{\partial u_{y}}+\frac{2 \alpha}{\alpha-1} F\right)=0 \\
& C_{2}\left(u_{x} \frac{\partial G}{\partial u_{x}}+u_{y} \frac{\partial G}{\partial u_{y}}+\frac{2 \alpha}{\alpha-1} G\right)=0 \\
& C_{2}\left(u_{x} \frac{\partial H}{\partial u_{x}}+u_{y} \frac{\partial H}{\partial u_{y}}+\frac{2 \alpha}{\alpha-1} H\right)=0
\end{aligned}
$$

а расщепление по $u_{x x}, u_{y y}, u_{z z}$ дает

$$
\begin{aligned}
\gamma_{1} \frac{\partial F}{\partial u_{x}}+\gamma_{2} \frac{\partial F}{\partial u_{y}} & =2 \theta_{x}^{1} F+\theta_{y}^{1} H-\alpha C_{1} F \\
\gamma_{1} \frac{\partial G}{\partial u_{x}}+\gamma_{2} \frac{\partial G}{\partial u_{y}} & =2 \theta_{y}^{2} G+\theta_{x}^{2} H-\alpha C_{1} G, \\
\gamma_{1} \frac{\partial H}{\partial u_{x}}+\gamma_{2} \frac{\partial H}{\partial u_{y}} & =2 \theta_{x}^{2} F+2 \theta_{y}^{1} G+\left(\theta_{x}^{1}+\theta_{y}^{2}\right) H-\alpha C_{1} H,
\end{aligned}
$$

где

$$
\begin{aligned}
& \gamma_{1} \equiv \gamma_{1}\left(x, y, u_{x}, u_{y}\right)=\psi_{x}+\left(\varphi-\theta_{x}^{1}\right) u_{x}-\theta_{x}^{2} u_{y} \\
& \gamma_{2} \equiv \gamma_{2}\left(x, y, u_{x}, u_{y}\right)=\psi_{y}-\theta_{y}^{1} u_{x}+\left(\varphi-\theta_{y}^{2}\right) u_{y}
\end{aligned}
$$

Оставшееся после указанных расщеплений уравнение имеет вид

$$
\begin{aligned}
{ }_{0} D_{t}^{\alpha}(\psi)=\left(\psi_{x x}+(\right. & \left.2 \varphi_{x}-\theta_{x x}^{1}\right) \\
& \left.u_{x}-\theta_{x x}^{2} u_{y}\right) F+ \\
& +\left(\psi_{y y}-\theta_{y y}^{1} u_{x}+\left(2 \varphi_{y}-\theta_{y y}^{2}\right) u_{y}\right) G+ \\
& +\left(\psi_{x y}+\left(\varphi_{y}-\theta_{x y}^{1}\right) u_{x}+\left(\varphi_{x}-\theta_{x y}^{2}\right) u_{y}\right) H .
\end{aligned}
$$

Из (21) следует, что если $C_{2} \neq 0$, то

$$
F=u_{x}^{2 \delta} \tilde{F}\left(\frac{u_{x}}{u_{y}}\right), \quad G=u_{x}^{2 \delta} \tilde{G}\left(\frac{u_{x}}{u_{y}}\right), \quad H=u_{x}^{2 \delta} \tilde{H}\left(\frac{u_{x}}{u_{y}}\right), \quad \delta=\frac{\alpha}{1-\alpha},
$$

где $\tilde{F}, \tilde{G}, \tilde{H}$ - произвольные функции. Подстановка этих представлений в (18) и решение получающихся в результате обыкновенных дифференциальных уравнений дает

$$
f(r)=f_{0} r^{\delta}, \quad g(r)=g_{0} r^{\delta}, \quad f_{0}, g_{0}=\text { const }, \quad r=u_{x}^{2}+u_{y}^{2}
$$


Если $C_{2}=0$, то $(21)$ выполнено тождественно. В этом случае подстановка (18) в (20) и решение получающихся в результате уравнений дает, что либо $f=f_{0}=$ const, $g=g_{0}=$ const, либо $\varphi_{x}=\varphi_{y}=0$, то есть $\varphi=C_{3}=$ const.

Дальнейшая классификация проводится на основе анализа системы (22) и уравнения (23), который показывает, что выделяются отдельно случай степенных функций $f(r)=f_{0} r^{\beta}, g(r)=g_{0} r^{\beta}\left(f_{0}, g_{0}=\mathrm{const}\right)$ и случай $f(r)=g(r)$. Окончательные результаты классификации формулируются в виде следующего утверждения.

Теорема 2. Уравнение (1) в случае произволъных функиий $f$ u $g$ имеет четырехмерную алгебру Ли симметрий с базисом

$$
X_{1}=\frac{\partial}{\partial x}, \quad X_{2}=\frac{\partial}{\partial y}, \quad X_{3}=\frac{2}{\alpha} t \frac{\partial}{\partial t}+x \frac{\partial}{\partial x}+y \frac{\partial}{\partial y}+u \frac{\partial}{\partial u}, \quad X_{4}=t^{\alpha-1} \frac{\partial}{\partial u} .
$$

$B$ cлучае $f(r)=f_{0} r^{\beta}, g(r)=g_{0} r^{\beta}, f_{0}, g_{0}=$ const, $\beta \neq 0, n p u f_{0} \neq g_{0}$ алгебра расширяется до пятимерной оператором группы неравномерных растяжений

$$
X_{5}=\beta x \frac{\partial}{\partial x}+\beta y \frac{\partial}{\partial y}+(1+\beta) u \frac{\partial}{\partial u} .
$$

В частном случае $\beta=\alpha /(1-\alpha)$ происходит дополнительное расширение алгебры до шестимерной оператором проективной группъ

$$
X_{6}=t^{2} \frac{\partial}{\partial t}+(\alpha-1) t u \frac{\partial}{\partial u}
$$

В изотропном случае $(f=g)$ размерности всех указанных выше алгебр увеличиваются на единицу за счет дополнительно допускаемого оператора группь вращенич

$$
X_{r}=y \frac{\partial}{\partial x}-x \frac{\partial}{\partial y} .
$$

При $\alpha \in(1,2)$ размерности всех приведеннъх алгебр дополнительно увеличиваются на единичу, а базис дополняется оператором

$$
X_{\alpha}=t^{\alpha} \frac{\partial}{\partial u} \text {. }
$$

Линейный случай $f=f_{0}, g=g_{0}, f_{0}, g_{0}=\mathrm{const,}$ растяжением пространственных переменных сводится $\kappa f_{0}=g_{0}=1$. В этом случае алгебра является бесконечномерной и ее базис образуют операторы $X_{1}, X_{2}, X_{3}$ из (24), оператор $X_{r}$ uз (27) и операторь

$$
X_{u}=u \frac{\partial}{\partial u}, \quad X_{\infty}=\psi \frac{\partial}{\partial u},
$$

где функиия $\psi(t, x, y)$ является произвольным решением линейного уравнения

$$
{ }_{0} D_{t}^{\alpha} u=u_{x x}+u_{y y}
$$

Основные отличия результатов теоремы 2 от аналогичных результатов групповой классификации классического ортотропного уравнения фильтрации (соответствующего $\alpha=1$ ) заключаются в следующем: 
1) дробно-дифференциальное уравнение фильтрации не допускает оператор группы переносов по временной переменной $t$, что является характерной особенностью всех дифференциальных уравнений с дробными производными по времени [18, 19];

2) выделяется особый вид степенной зависимости коэффициентов пьезопроводности, не имеющий аналога в классическом уравнении фильтрации, при котором уравнение допускает оператор проективной группы $X_{6}$;

3) операторы групп растяжений $X_{3}$ и $X_{5}$ оказываются зависящими от порядка дробного дифференцирования $\alpha$.

Также от него зависят операторы $X_{4}$ и $X_{\alpha}$, соответствующие сдвигу $u$ на частное решение дробно-дифференциального уравнения.

Полученная в теореме 2 групповая классификация уравнения (1) может быть использована для нахождения его инвариантно-групповых решений и построения законов сохранения.

3. Представление инвариантных решений. Рассмотрим задачу построения представлений инвариантно-групповых решений для нелинейного ортотропного уравнения (1) по допускаемым им неподобным двумерным подалгебрам алгебр Ли симметрий.

В случае произвольных функций $f$ и $g(f \neq g)$ уравнение допускает четырехмерную алгебру Ли симметрий (см. теорему 2) с базисом (24). Данная алгебра изоморфна алгебре $A_{4,5}^{a, a}$ (по классификации [29]) с ненулевыми коммутационными соотношениями

$$
\left[e_{1}, e_{4}\right]=e_{1}, \quad\left[e_{2}, e_{4}\right]=a e_{2}, \quad\left[e_{3}, e_{4}\right]=a e_{3}, \quad-1<b<1,
$$

в которую она переходит в результате замены базиса

$$
e_{1}=X_{3}, \quad e_{2}=X_{1}, \quad e_{3}=X_{2}, \quad e_{4}=b X_{4}, \quad b=\frac{\alpha}{2-\alpha} .
$$

Построим неподобные инвариантно-групповые решения уравнения (1) на основе оптимальной системы двумерных подалгебр алгебры $A_{4,5}^{a, a}$, построенной в [29]:

1) $\left\{e_{1}+\varepsilon e_{2}, e_{3}\right\}$,

2) $\left\{e_{1}+\varepsilon e_{3}, e_{2}+\beta e_{3}\right\}$,

4) $\left\{e_{1}, e_{2} \cos \theta+e_{3} \sin \theta\right\}$,

3) $\left\{e_{2}, e_{3}\right\}$,

5) $\left\{e_{1}, e_{4}\right\}$,

6) $\left\{e_{2} \cos \theta+e_{3} \sin \theta, e_{4}\right\}$,

где $\theta \in[0, \pi), \varepsilon= \pm 1, \beta \in \mathbb{R}$.

Подалгебры 5) и 6) не позволяют построить инвариантное решение, так как не удовлетворяют соответствующему необходимому условию его существования (см., например, [13]). Вычисление инвариантов остальных подалгебр приводит к следующим анзацам инвариантных решений:

1) $u=\varepsilon t^{\alpha-1} x+\varphi(t)$,

2) $u=-\varepsilon \beta t^{\alpha-1} x+t^{\alpha-1} y+\varphi(t)$,

3) $u=\varphi(t)$,

4) $u=(y \cos \theta-x \sin \theta) \varphi(\xi), \quad \xi=\frac{t}{|y \cos \theta-x \sin \theta|^{\frac{2}{\alpha}}}$, 
где $\varphi(z)$ - новая искомая функция одного аргумента. Подстановка этих анзацев в исходное уравнение сводит задачу нахождения инвариантного решения к решению так называемых редуцированных уравнений (или факторуравнений), представляющих собой в данном случае обыкновенное дробнодифференциальное уравнение порядка $\alpha$. В первых трех случаях это уравнение имеет вид

$$
{ }_{0} D_{t}^{\alpha} \varphi=0
$$

его общее решение хорошо известно $[1,2]$ :

$$
\varphi(t)=C t^{\alpha-1}
$$

где $C$ - произвольная постоянная. В результате приходим к следующим весьма простым неподобным инвариантным решениям:

$$
\begin{aligned}
& \text { 1) } u=C t^{\alpha-1}, \\
& \text { 2) } u=t^{\alpha-1}(\varepsilon x+C), \\
& \text { 3) } u=t^{\alpha-1}(\varepsilon \beta x+y+C), \quad \beta \in \mathbb{R}, \quad \varepsilon= \pm 1 .
\end{aligned}
$$

Более интересным оказывается случай 4), для которого редуцированное уравнение имеет вид

$$
{ }_{0} D_{\xi}^{\alpha} \varphi+\frac{2}{\alpha} \xi\left[\sin ^{2} \theta(\psi f(\psi))_{\xi}+\cos ^{2} \theta(\psi g(\psi))_{\xi}\right]=0,
$$

где $\psi(\xi)=\varphi(\xi)-\frac{2}{\alpha} \xi \varphi^{\prime}(\xi)$.

Уравнение (28) в общем случае аналитически решено быть не может, поэтому рассмотрим некоторые его частные случаи.

Пусть $f=1$ и $g(\psi)=\psi^{-1}$. Тогда уравнение $(28)$ переходит в линейное уравнение

$$
\alpha_{0}^{2} D_{\xi}^{\alpha} \varphi=4 \xi^{2} \sin ^{2} \theta \varphi^{\prime \prime}+2(2-\alpha) \sin ^{2} \theta \xi \varphi^{\prime} .
$$

При $\theta=0$ решение уравнения имеет вид $\varphi(\xi)=C \xi^{\alpha-1}$, что дает инвариантное решение исходного уравнения

$$
u=C t^{\alpha-1} y^{\frac{2}{\alpha}-1} .
$$

При $\theta \neq 0$ заменой переменных $\tau=\xi(\sin \theta)^{\frac{2}{\alpha}}, z(\tau)=\varphi(\xi(\tau))$ уравнение (28) приводится к виду

$$
\alpha_{0}^{2} D_{\tau}^{\alpha} z=4 \tau^{2} z^{\prime \prime}+2(2-\alpha) \tau z^{\prime},
$$

который является частным случаем уравнения, рассмотренного в работе [30], и имеет общее решение

$$
z(\tau)=\tau^{-\frac{\alpha}{2}}\left[C_{1} \phi\left(-\frac{\alpha}{2}, 1+\frac{\alpha}{2} ; \tau^{-\frac{\alpha}{2}}\right)+C_{2} \phi\left(-\frac{\alpha}{2}, 1+\frac{\alpha}{2} ;-\tau^{-\frac{\alpha}{2}}\right)\right] .
$$

Здесь $C_{1}$ и $C_{2}$ - произвольные постоянные,

$$
\phi(\rho, \mu ; z)=\sum_{k=0}^{\infty} \frac{1}{\Gamma(\rho k+\mu)} \frac{z^{k}}{k !}, \quad \rho>0, \quad \mu, z \in \mathbb{C}
$$


- функция Райта. Тогда инвариантное решение имеет вид

$$
u=(y \cos \theta-x \sin \theta) z\left(\frac{t(\sin \theta)^{\frac{2}{\alpha}}}{|y \cos \theta-x \sin \theta|^{\frac{2}{\alpha}}}\right) .
$$

Теперь рассмотрим случай $f(\psi)=\psi^{\beta-1}(\beta \neq 0,1), g(\psi)=\psi^{-1}$. Тогда уравнение (28) принимает вид

$$
\alpha_{0}^{\beta+1} D_{\xi}^{\alpha} \varphi+2 \beta \sin ^{2} \theta \xi\left(\alpha \varphi-2 \xi \varphi^{\prime}\right)^{\beta-1}\left((\alpha-2) \varphi^{\prime}-2 \xi \varphi^{\prime \prime}\right)=0 .
$$

Данное уравнение при $\alpha<\beta<1$ имеет частное степенное решение

$$
\varphi(\xi)=A \xi^{\gamma}, \quad A=\left[\frac{\alpha^{\beta+1}}{2 \beta \sin ^{2} \theta} \frac{\Gamma(\gamma)}{\Gamma(\gamma+1-\alpha)}\right]^{\frac{1}{\beta-1}}, \quad \gamma=\frac{1-\alpha}{\beta-1} .
$$

Соответствующее инвариантное решение исходного уравнения имеет вид

$$
u=A t^{\gamma} \frac{(y \cos \theta-x \sin \theta)}{|y \cos \theta-x \sin \theta|^{\frac{2}{\alpha} \gamma}} .
$$

Теперь рассмотрим случай $f(r)=f_{0} r^{\beta}, g(r)=g_{0} r^{\beta}, f_{0}, g_{0}=\operatorname{const}\left(f_{0} \neq g_{0}\right)$, $\beta \neq 0$. В этом случае алгебра Ли симметрий $L_{5}$ является пятимерной с базисом $X_{1}, \ldots, X_{5}$, определяемым (24), (25). Соответствующая оптимальная система подалгебр строится на основе двухшагового алгоритма, предложенного академиком Л. В. Овсянниковым в работе [31] (см. также [32]). Базисы найденных неподобных двумерных подалгебр из оптимальной системы $\Theta_{2} L_{5}$ и соответствующие представления инвариантных решений с одной независимой переменной приведены в табл. 1. В таблице для подалгебр используется сокращенная форма записи: например, $\Theta, p 2+4$ означает двумерную подалгебру с базисом из операторов $X_{1} \sin \theta+X_{2} \cos \theta, p X_{2}+X_{4}$. В табл. 2 представлены аналогичные результаты для шестимерной алгебры $L_{6}$ с базисом $X_{1}, \ldots, X_{6}$, соответствующей частному случаю $\beta=\alpha /(1-\alpha)$.

Все анзацы инвариантных решений записаны в табл. 1 и 2 таким образом, чтобы после симметрийной редукции соответствующее редуцированное уравнение относительно функции $\varphi(z)$ было либо обыкновенным дифференциальным уравнением целого порядка (если $z$ не зависит от $t$ ), либо обыкновенным дробно-дифференциальным уравнением с дробной производной Римана-Лиувилля по переменной $z$. Замены переменных, позволяющие сохранить тип оператора дробного дифференцирования при симметрийной редукции, приведены в [30].

4. Построение законов сохранения. Уравнение (1) не обладает классическим лагранжианом, поэтому для построения его законов сохранения с использованием симметрий может быть применен принцип нелинейной самосопряженности, изначально предложенный профессором Н. Х. Ибрагимовым для дифференциальных уравнений целого порядка $[27,28]$ и обобщенный в работах $[33,34]$ на дифференциальные уравнения дробного порядка.

Уравнение (1) может быть представлено в виде

$$
\mathscr{F}\left(t, x, y, u, u_{x}, u_{y}, u_{x x}, u_{x y}, u_{y y},{ }_{0} D_{t}^{\alpha} u\right)=0 .
$$


Таблица 1

Представления инвариантных решений для алгебры $L_{5}$

[Representations of invariant solutions for algebra $L_{5}$ ]

\begin{tabular}{|c|c|c|}
\hline no. & a subalgebra of $\Theta_{2} L_{5}$ & $u(t, x, y)$ \\
\hline 1 & 1,2 & $\varphi(t)$ \\
\hline 2 & $2, p 1+4$ & $p^{-1} x t^{\alpha-1}+\varphi(t) \quad(p \neq 0)$ \\
\hline 3 & 3,5 & $x^{\gamma} t^{\alpha(1-\gamma) / 2} \varphi(y / x)$ \\
\hline 4 & $2,1+3-5$ & $e^{(1-\gamma) x} \varphi\left(t e^{-2 x / \alpha}\right)$ \\
\hline 5 & $\Theta, p 2+4$ & $p^{-1} t^{\alpha-1}(x \operatorname{ctg} \theta-y)+\varphi(t) \quad(p, \theta \neq 0)$ \\
\hline 6 & $\Theta, 5$ & $(x \cos \theta-y \sin \theta)^{\gamma} \varphi(t)$ \\
\hline 7 & $\Theta, 3+r 5$ & $\begin{array}{c}z^{1+p^{\gamma}} \varphi\left(t z^{-2 /[\alpha(p+1)]}\right) \quad(r \neq-1) \\
t^{\alpha(1-\gamma) / 2} \varphi(z)(r=-1) \\
z=(x \cos \theta-y \sin \theta)\end{array}$ \\
\hline 8 & $\Theta, 2+3-5$ & $\begin{array}{c}z^{1-\gamma} \varphi\left(t z^{-2 / \alpha}\right), \quad z=e^{y-x \operatorname{ctg} \theta} \quad(\theta \neq 0) \\
t^{\alpha(1-\gamma) / 2} \varphi(x)(\theta=0)\end{array}$ \\
\hline 9 & $\begin{array}{c}\Theta, 3+4-\frac{a}{\gamma} 5(\gamma \neq 0) \\
\Theta, 4+5(\gamma=0)\end{array}$ & $\begin{array}{c}z^{1-a} \varphi\left(t z^{-2 / \alpha}\right), \quad z=(x \cos \theta-y \sin \theta)^{1-a / \gamma} \quad(\gamma \neq a) \\
t^{\alpha-1}[(\alpha / 2) \ln t+\varphi(x \cos \theta-y \sin \theta)] \quad(\gamma=a) \\
\varphi(t)+t^{\alpha-1} \ln |x \cos \theta-y \sin \theta|\end{array}$ \\
\hline 10 & $p 1+q 2+4,3+\frac{1-a}{\gamma-1} 5$ & $\begin{array}{c}u=p^{-1} x t^{\alpha-1}+z^{2 \gamma-1-a \gamma} \varphi\left(t z^{2(1-\gamma) / \alpha}\right) \\
z=(q x-p y)^{1 /(\gamma-a)} \quad(\gamma \neq a, p \neq 0) \\
q^{-1} t^{\alpha-1} y+z^{2 \gamma-1-a \gamma} \varphi\left(t z^{1-\gamma}\right) \\
z=x^{1 /(\gamma-a)} \quad(\gamma \neq a, p=0, q \neq 0) \\
p^{-1} t^{\alpha-1}[x+\varphi(q x-p y)] \quad(\gamma=a, p \neq 0) \\
q^{-1} t^{\alpha-1}[y+\varphi(x)] \quad(\gamma=a, p=0, q \neq 0)\end{array}$ \\
\hline 11 & $\begin{array}{l}\Theta, p 2+3+4-5 \\
(\gamma=a, p \neq 0)\end{array}$ & $\begin{array}{c}z^{a-1} \varphi\left(t z^{-2 / \alpha)}, \quad z=e^{(y-x \operatorname{ctg} \theta) / p}(\theta \neq 0)\right. \\
t^{\alpha-1}[(\alpha / 2) \ln t+\varphi(x)](\theta=0)\end{array}$ \\
\hline 12 & $\begin{array}{l}X_{2}, p 1+3+4-5 \\
\quad(\gamma=a, p \neq 0)\end{array}$ & $t^{\alpha-1}\left[(\alpha / 2) \ln t+\varphi\left(t e^{-2 x /(\alpha p)}\right)\right]$ \\
\hline 13 & $\begin{array}{l}p 1+q 2+4, \Theta+3-5 \\
(\gamma=a)\end{array}$ & 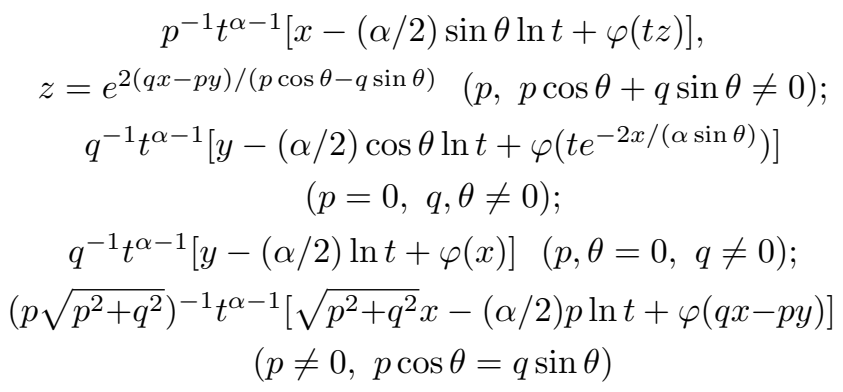 \\
\hline
\end{tabular}

Here are $\Theta=X_{1} \sin \theta+X_{2} \cos \theta, \theta \in\left[0, \frac{\pi}{2}\right], \gamma=1+\beta^{-1}, a=2 \alpha^{-1}-1, p, q \geqslant 0, r \in \mathbb{R}$. 
Таблица 2

Представления инвариантных решений для алгебры $L_{6}$

[Representations of invariant solutions for algebra $L_{6}$ ]

\begin{tabular}{c||c|c}
\hline no. & a subalgebra of $\Theta_{2} L_{6}$ & $u(t, x, y)$ \\
\hline $1-10$ & are identical as $1-10$ & are identical as $1-10$ from \\
& from Table 1 & Table 1 \\
11 & 5,6 & $t^{\alpha-1} x^{1 / \alpha} \varphi(y / x)$ \\
12 & $3+p 5,6$ & $t^{\alpha-1} x^{(2+p-\alpha) /(\alpha(p+1))} \varphi(y / x)(p \neq-1)$ \\
13 & $\Theta, 6$ & $t^{\alpha-1} \varphi(x \cos \theta-y \sin \theta)$ \\
14 & $\Theta, 5+\varepsilon 6$ & $t^{\alpha-1} z^{1 / \alpha} \varphi\left(\frac{t}{\varepsilon+t \ln z}\right), z=x \cos \theta-y \sin \theta$ \\
15 & $\Theta, 4+6$ & $t^{\alpha-1} \varphi(x \cos \theta-y \sin \theta)$ \\
16 & $\Theta, 2+p 4+6(\theta \neq 0)$ & $t^{\alpha-1} \varphi\left(\frac{t}{1+t z}\right), z=y-x \operatorname{ctg} \theta(p=0)$ \\
17 & $2,1+p 4+6$ & $t^{\alpha-1} \varphi\left(\frac{t}{1+t x}\right)(p=0)$ \\
18 & $p 1+q 2+4,6$ & $q^{-1} t^{\alpha-1}[y+\varphi(p y-q x)](q \neq 0)$ \\
19 & $p 1+q 2+4,1+6$ & $p^{-1} t^{\alpha-1}[x+\varphi(y)](p \neq 0)$ \\
20 & $p 1+q 2+4, r 1+2+6$ & $q^{-1} t^{\alpha-1}\left[y+\varphi\left(\frac{t}{q+t(q x-p y)}\right)\right](q \neq 0)$ \\
& & $p^{-1} t^{\alpha-1}\left[x+t^{-1}+\varphi(y)\right](p \neq 0)$ \\
& & $t^{\alpha-1}\left[y+t^{-1}+\varphi\left(\frac{t}{q r-p+t(q x-p y)}\right)\right](q \neq 0)$ \\
& & $p^{-1} t^{\alpha-1}\left[x-r y+\varphi\left(\frac{t}{1+t y}\right)\right](p \neq 0)$
\end{tabular}

Here are $\Theta=X_{1} \sin \theta+X_{2} \cos \theta, \theta \in\left[0, \frac{\pi}{2}\right], p, q \geqslant 0, r \in \mathbb{R}$.

Для него вводится формальный лагранжиан вида $\mathscr{L}=v \mathscr{F}$, где $v=v(t, x, y)-$ новая зависимая переменная. Тогда уравнение, сопряженное к (1), находится каK

$$
\frac{\delta \mathscr{L}}{\delta u}=0
$$

где вариационная производная имеет в данном случае вид

$$
\frac{\delta}{\delta u}=-D_{x} \frac{\partial}{\partial u_{x}}-D_{y} \frac{\partial}{\partial u_{y}}+D_{x}^{2} \frac{\partial}{\partial u_{x x}}+D_{y}^{2} \frac{\partial}{\partial u_{y y}}+D_{x} D_{y} \frac{\partial}{\partial u_{x y}}+{ }_{t}^{C} D_{T}^{\alpha} \frac{\partial}{\partial_{0} D_{t}^{\alpha} u} .
$$

Здесь ${ }_{t}^{C} D_{T}^{\alpha}$ - оператор правостороннего дробного дифференцирования Герасимова-Капуто порядка $\alpha$, определенный для $t \in(0, T)[2,7]$ :

$$
{ }_{t}^{C} D_{T}^{\alpha} u=\frac{(-1)^{n}}{\Gamma(n-\alpha)} \int_{t}^{T} \frac{1}{(\tau-t)^{\alpha-n+1}} \frac{\partial^{n} u(\tau, x, y)}{\partial \tau^{n}} d \tau, \quad n=[\alpha]+1 .
$$

Для уравнения (1), представленного в форме (17), вычисления приводят к следующему сопряженному уравнению:

$$
{ }_{t}^{C} D_{T}^{\alpha} v=-D_{x}\left[v\left(\frac{\partial F}{\partial u_{x}} u_{x x}+\frac{\partial G}{\partial u_{x}} u_{y y}+\frac{\partial H}{\partial u_{x}} u_{x y}\right)\right]-
$$




$$
\begin{aligned}
& -D_{y}\left[v\left(\frac{\partial F}{\partial u_{y}} u_{x x}+\frac{\partial G}{\partial u_{y}} u_{y y}+\frac{\partial H}{\partial u_{y}} u_{x y}\right)\right]+v_{x x} F+2 v_{x} D_{x} F+v D_{x}^{2} F+ \\
& +v_{y y} G+2 v_{y} D_{y} G+v D_{y}^{2} G+v_{x y} H+v_{x} D_{y} H+v_{y} D_{x} H+v D_{x} D_{y} H .
\end{aligned}
$$

Если найдется такая подстановка $v=\varphi(t, x, y, u)$, что приведенное сопряженное уравнение будет выполнено тождественно на всех решениях $u(t, x, y)$ исходного уравнения (1), то уравнение (1) называется нелинейно самосопряженным. В [28] доказано, что любое линейное уравнение является нелинейно самосопряженным. Поэтому в линейном случае $f=f_{0}=$ const, $g=g_{0}=$ const уравнение (1) является нелинейно самосопряженным с подстановкой $v=$ $=\psi(t, x, y)$, где $\psi(t, x, y)$ - любое решение его сопряженного уравнения (29), которое в данном случае также будет линейным. Для нелинейного случая в результате вычислений получено, что $\varphi=\varphi(x, y)$, и эта функция должна удовлетворять следующей системе уравнений:

$$
\begin{aligned}
& \varphi_{x} \frac{\partial F}{\partial u_{x}}+\varphi_{y}\left(\frac{\partial H}{\partial u_{x}}-\frac{\partial F}{\partial u_{y}}\right)=0, \\
& \varphi_{x}\left(\frac{\partial H}{\partial u_{y}}-\frac{\partial G}{\partial u_{x}}\right)+\varphi_{y} \frac{\partial G}{\partial u_{y}}=0, \\
& \varphi_{x} \frac{\partial F}{\partial u_{y}}+\varphi_{y} \frac{\partial G}{\partial u_{x}}=0 \\
& \varphi_{x x} F+\varphi_{y y} G+\varphi_{x y} H=0 .
\end{aligned}
$$

Для произвольных функций $f$ и $g$ единственным решением этой системы будет $\varphi=c$, где $c$ - произвольная постоянная. Также выделяются два особых случая:

1) $f$ - произвольная функция, $g=$ const, тогда $\varphi=c_{1}+c_{2} y$;

2) $g$ - произвольная функция, $f=$ const, тогда $\varphi=c_{1}+c_{2} x$.

Таким образом, доказана

Теорема 3. Уравнение (1) является нелинейно самосопряжсенным. Соответствующая подстановка $v=\varphi(t, x, y, u)$, обращающая уравнение (29) в тождество на всех решениях уравнения (1), имеет следующий вид:

1) $v=c$ в случае произвольных функиий $f$ u $g$, при этом формальныи лагранжиан $\mathscr{L}$ совпадает с самим уравнением (1);

2) $v=c_{1}+c_{2} y$ в случае, когда функиия $f$ произвольная, a $g=$ const;

3) $v=c_{1}+c_{2} x$ в случае, когда бункиия $g$ произвольная, a $f=$ const;

4) $v=\psi(t, x, y)$, где $\psi(t, x, y)$ - любое решение линейного сопряженного уравнения (29) при $f=$ const, $g=$ const.

Здесь с, $c_{1}, c_{2}$ - произвольные постоянные.

Поскольку уравнение (1) является нелинейно самосопряженным, по любой его известной симметрии вида (3) может быть найден соответствующий закон сохранения

$$
D_{t} C^{t}+D_{x} C^{x}+D_{y} C^{y}=0
$$

Координаты сохраняющегося вектора $\left(C^{t}, C^{x}, C^{y}\right)$ будут при этом находиться 
по следующим формулам (см. [19,35]):

$$
\begin{aligned}
& C^{t}={ }_{0} I_{t}^{1-\alpha} W \frac{\partial \mathscr{L}}{\partial_{0} D_{t}^{\alpha} u}+J\left\{W, D_{t}\left(\frac{\partial \mathscr{L}}{\partial_{0} D_{t}^{\alpha} u}\right)\right\}, \\
& C^{x}=W\left(\frac{\partial \mathscr{L}}{\partial u_{x}}-D_{x} \frac{\partial \mathscr{L}}{\partial u_{x x}}-D_{y} \frac{\partial \mathscr{L}}{\partial u_{x y}}\right)+D_{x}(W) \frac{\partial \mathscr{L}}{\partial u_{x x}}+D_{y}(W) \frac{\partial \mathscr{L}}{\partial u_{x y}}, \\
& C^{y}=W\left(\frac{\partial \mathscr{L}}{\partial u_{y}}-D_{x} \frac{\partial \mathscr{L}}{\partial u_{y x}}-D_{y} \frac{\partial \mathscr{L}}{\partial u_{y y}}\right)+D_{x}(W) \frac{\partial \mathscr{L}}{\partial u_{y x}}+D_{y}(W) \frac{\partial \mathscr{L}}{\partial u_{y y}},
\end{aligned}
$$

где $W=\eta-\tau u_{t}-\xi^{1} u_{x}-\xi^{2} u_{y}$

$$
J\{f, g\}=\frac{1}{\Gamma(1-\alpha)} \int_{0}^{t} \int_{t}^{T} \frac{f(\tau, x, y) g(\mu, x, y)}{(\mu-\tau)^{\alpha}} d \mu d \tau
$$

В случае произвольных $f$ и $g$ подстановка в эти формулы формального лагранжиана

$$
\mathscr{L}={ }_{0} D_{t}^{\alpha} u-F\left(u_{x}, u_{y}\right) u_{x x}-G\left(u_{x}, u_{y}\right) u_{y y}-\frac{1}{2} H\left(u_{x}, u_{y}\right) u_{x y}-\frac{1}{2} H\left(u_{x}, u_{y}\right) u_{y x}
$$

где функции $F, G, H$ определены в (18), дает

$$
\begin{gathered}
C^{t}={ }_{0} I_{t}^{1-\alpha} W \\
C^{x}=W\left[2 u_{x} u_{y}\left(u_{x} u_{x y}+u_{y} u_{y y}\right)\left(f^{\prime \prime}-g^{\prime \prime}\right)+\left(u_{y} u_{x y}+u_{x} u_{y y}\right)\left(f^{\prime}-g^{\prime}\right)\right]- \\
-\left(f+2 u_{x}^{2} f^{\prime}\right) D_{x}(W)-u_{x} u_{y}\left(f^{\prime}+g^{\prime}\right) D_{y}(W), \\
C^{y}=W\left[2 u_{x} u_{y}\left(u_{y} u_{x y}+u_{x} u_{x x}\right)\left(g^{\prime \prime}-f^{\prime \prime}\right)+\left(u_{x} u_{x y}+u_{y} u_{x x}\right)\left(g^{\prime}-f^{\prime}\right)\right]- \\
-u_{x} u_{y}\left(f^{\prime}+g^{\prime}\right) D_{x}(W)-\left(g+2 u_{y}^{2} g^{\prime}\right) D_{y}(W) .
\end{gathered}
$$

Операторам (24) соответствуют следующие значения $W$ :

$$
W_{1}=-u_{x}, \quad W_{2}=-u_{y}, \quad W_{3}=u-\frac{2}{\alpha} t u_{t}-x u_{x}-y u_{y}, \quad W_{4}=t^{\alpha-1} .
$$

Легко видеть, что оператор $X_{4}$ дает тривиальный закон сохранения с $C^{t}=0$, а операторы $X_{1}$ и $X_{2}$ дают законы сохранения, сводящиеся к тривиальному элементарными преобразованиями с учетом самого уравнения (1). Единственный нетривиальный закон сохранения порождается оператором $X_{3}$, соответствующий сохраняющийся вектор имеет координаты

$$
C^{t}={ }_{0} I_{t}^{1-\alpha} u, \quad C^{x}=-f\left(u_{x}^{2}+u_{y}^{2}\right) u_{x}, \quad C^{y}=-g\left(u_{x}^{2}+u_{y}^{2}\right) u_{y} .
$$

Данный закон сохранения соответствует исходному уравнению (1). Других нетривиальных законов сохранения в случае произвольных функций $f$ и $g$ метод нелинейной самосопряженности с подстановкой вида $v=\varphi(t, x, y, u)$ для уравнения (1) не дает. 
В случае $f(r)=f_{0} r^{\beta}, g(r)=g_{0} r^{\beta}$ оператор $X_{5}$ из (25) также порождает закон сохранения с сохраняющимся вектором (30). При $\beta=\alpha /(1-\alpha)$ оператор $X_{6}$ из (26) порождает новый закон сохранения, координаты сохраняющегося вектора которого имеют вид

$$
C^{t}=t_{0} I_{t}^{1-\alpha} u-{ }_{0} I_{t}^{2-\alpha} u, \quad C^{x}=-f_{0} t u_{x}\left(u_{x}^{2}+u_{y}^{2}\right)^{\frac{\alpha}{1-\alpha}}, \quad C^{y}=-g_{0} t u_{y}\left(u_{x}^{2}+u_{y}^{2}\right)^{\frac{\alpha}{1-\alpha}}
$$

В случае $f=g$ оператор $X_{r}$ из $(27)$ также порождает лишь тривиальный закон сохранения.

Более интересными представляются случаи 2) и 3) из теоремы 3. Для произвольной функции $f$ и $g=g_{0}=$ const при $v=c_{1}+c_{2} y$ для операторов $X_{1}$ и $X_{2}$ получаем закон сохранения с сохраняющимся вектором (30). Оператор $X_{3}$ порождает в этом случае новый закон сохранения:

$$
C^{t}=y_{0} I_{t}^{1-\alpha} u, \quad C^{x}=-y u_{x} f\left(u_{x}^{2}+u_{y}^{2}\right), \quad C^{y}=g_{0}\left(u-y u_{y}\right) .
$$

Аналогично, для случая $f=f_{0}=$ const и произвольной функции $g$ оператор $X_{3}$ при $v=c_{1}+c_{2} x$ дает

$$
C^{t}=x_{0} I_{t}^{1-\alpha} u, \quad C^{x}=f_{0}\left(u-x u_{x}\right), \quad C^{y}=-x u_{y} g\left(u_{x}^{2}+u_{y}^{2}\right) .
$$

Других законов сохранения для нелинейного уравнения (1) метод нелинейной самосопряженности с подстановкой вида $v=\varphi(t, x, y, u)$ не дает.

Найденные законы сохранения могут быть использованы, в частности, для построения частных решений уравнения (1) по методу, предложенному В [36].

Конкурирующие интересы. Авторы заявляют об отсутствии конкурирующих интересов.

Авторский вклад и ответственность. Все авторы принимали участие в получении основных результатов статьи и в написании рукописи. Авторы несут полную ответственность за предоставление окончательной рукописи в печать. Окончательная версия рукописи была одобрена всеми авторами.

Финансирование. Работа выполнена в рамках государственного задания Министерства науки и высшего образования РФ (проект № 1.3103.2017/4.6).

\section{Библиографический список}

1. Samko S. G., Kilbas A. A., Marichev O. I. Fractional integrals and derivatives. Theory and applications. New York: Gordon \& Breach Sci. Publishers, 1993. xxxvi+976 pp.

2. Kilbas A. A., Srivastava H. M., Trujillo J. J. Theory and Applications of Fractional Differential Equations / North-Holland Mathematics Studies. vol. 204. Amsterdam: Elsevier, 2006. $\mathrm{xv}+523 \mathrm{pp}$.

3. Podlubny I. Fractional differential equations. An introduction to fractional derivatives, fractional differential equations, to methods of their solution and some of their applications / Mathematics in Science and Engineering. vol. 198. San Diego: Academic Press, 1999. xxiv +340 pp.

4. Metzler R., Klafter J. The random walk's guide to anomalous diffusion: a fractional dynamic approach // Phys. Rep., 2000. vol. 339, no. 1. pp. 1-77. doi : 10.1016/S0370-1573(00) 00070-3.

5. Hilfer R. Applications of fractional calculus in physics. Singapore: World Scientific, 2000. vii $+463 \mathrm{pp}$. 
6. Нахушев А. М. Дробное исчисление и его применение. М.: Физматлит, 2003. 272 с.

7. Учайкин В. В. Метод дробных производнъх. Ульяновск: Артишок, 2008. 512 с.

8. Mainardi F. Fractional calculus and waves in linear viscoelasticity. An introduction to mathematical models. Hackensack: World Scientific, 2010. xx+347 pp.

9. Головизнин В. М., Кондратенко П. С., Матвеев Л. В. и др. Аномальная дифбузия радионуклидов в сильнонеоднородных геологических формациях. М.: Наука, 2010. 342 с.

10. Fractional Dynamics: Recent Advances / eds. J. Klafter, S. C. Lim, R. Metzler. Hackensack: World Scientific, 2011. xiv+515 pp.

11. Fractional kinetics in solids: Anomalous charge transport in semiconductors, dielectrics and nanosystems / eds. V. Uchaikin, R. Sibatov. Boca Raton: CRC Press, 2013. xvi+257 pp. doi: $10.1142 / 8185$.

12. Baleanu D., Diethelm K., Scalas E., Trujillo J. J. Fractional calculus: models and numerical methods / Series on Complexity, Nonlinearity and Chaos. vol. 5. Hackensack: World Scientific, 2017. xxviii+448 pp. doi: 10.1142/10044.

13. Ovsyannikov L. V. Group analysis of differential equations. New York: Academic Press, 1982. xvi+416 pp.

14. Olver P. J. Applications of Lie groups to differential equations / Graduate Texts in Mathematics. vol. 107. New York: Springer, 2000. xxviii +513 pp.

15. Bluman G. W., Cheviakov A. F., Anco S. C. Applications of symmetry methods to partial differential equations / Applied Mathematical Sciences. vol. 168. New York: Springer, 2010. xix +398 pp pp.. doi : 10.1007/978-0-387-68028-6.

16. Grigoriev Yu. N., Ibragimov N. H., Kovalev V. F., Meleshko S. V. Symmetries of integro-differential equations. With applications in mechanics and plasma physics./ Lecture Notes in Physics. vol. 806. Dordrecht: Springer, 2010. xiii +305 pp. doi: 10.1007/ 978-90-481-3797-8.

17. Ibragimov N. H. Transformation groups and Lie algebras. Hackensack: World Scientific, 2013. $\mathrm{x}+185$ pp. doi: $10.1142 / 8763$.

18. Gazizov R. K., Kasatkin A. A., Lukashchuk S. Y. Symmetries and group invariant solutions of fractional ordinary differential equations / Handbook of Fractional Calculus with Applications, Vol. 2 Fractional Differential Equations; eds. A. Kochubei, Y. Luchko. Berlin: De Gruyter, 2019. pp. 65-90. doi: 10.1515/9783110571660-004.

19. Gazizov R. K., Kasatkin A. A., Lukashchuk S. Y. Symmetries, conservation laws and group invariant solutions of fractional PDEs / Handbook of Fractional Calculus with Applications, Vol. 2 Fractional Differential Equations; eds. A. Kochubei, Y. Luchko. Berlin: De Gruyter, 2019. pp. 353-382. doi : 10.1515/9783110571660-016.

20. Raghavan R., Chen C. Fractional diffusion in rocks produced by horizontal wells with multiple, transverse hydraulic fractures of finite conductivity // J. Petrol. Sci. Eng., 2013. vol. 109. pp. 133-143. doi: 10.1016/j.petrol.2013.08.027.

21. Obembe A. D. Al-Yousef H. Y., Hossain M. E., Abu-Khamsin S. A. Fractional derivatives and their applications in reservoir engineering problems: A review // J. Petrol. Sci. Eng., 2017. vol. 157. pp. 312-327. doi: 10.1016/j . petrol.2017.07.035.

22. Газизов Р. К., Лукащук С. Ю. Дробно-дифференциальный подход к моделированию процессов фильтрации в сложных неоднородных пористых средах // Вестник УГАТУ, 2017. T. 21, № 4. С. 104-112.

23. Бабков О. К. О групповой классификации некоторых эволюционных уравнений / Teзисы международной конференции Mogran-16 (28 октября - 2 ноября 2013 г.). Уфа: УГАТУ, 2013. С. 6-7.

24. Овсянников Л. В. О свойстве $x$-автономии // Докл. РАН, 1993. Т. 330, № 5. С. 559-561.

25. Чиркунов Ю. А. Условия линейной автономности основной алгебры Ли системы линейных дифференциальных уравнений // Докл. РАН, 2009. Т. 426, № 5. С. 605-607.

26. Газизов Р. К., Касаткин А. А., Лукащук С. Ю. Уравнения с производными дробного порядка: замены переменных и нелокальные симметрии // Уфимск. матем. журн., 2012. T. 4, № 4. C. 54-68. 
27. Ibragimov N. H. A new conservation theorem // J. Math. Anal. Appl., 2007. vol. 333, no. 1. pp. 311-328. doi: 10.1016/j.jmaa.2006.10.078.

28. Ibragimov N. H. Nonlinear self-adjointness and conservation laws // J. Phys. A: Math. Theor., 2011. vol. 44, 432002. doi: 10.1088/1751-8113/44/43/432002.

29. Patera J., Winternitz P. Subalgebras of real three- and four-dimensional Lie algebras // J. Math. Phys., 1977. vol. 18, no.7. pp. 1449-1455. doi: 10.1063/1.523441.

30. Лукащук С. Ю. Симметрийная редукция и инвариантные решения нелинейного дробно-дифференциального уравнения аномальной диффузии с источником// Уфимск. матем. журн., 2016. Т. 8, № 4. С. 114-126.

31. Овсянников Л. В. Об оптимальных системах подалгебр // Докл. РАН, 1993. Т. 333, № 6. C. $702-704$.

32. Чиркунов Ю. А., Хабиров С. В. Элементы симметрийного анализа дифференциальных уравнений механики сплошной среды. Новосибирск: НГТУ, 2012. 659 с.

33. Lukashchuk S. Yu. Conservation laws for time-fractional subdiffusion and diffusionwave equations // Nonlinear Dyn., 2015. vol.80, no.1-2. pp. 791-802. doi:10.1007/ s11071-015-1906-7.

34. Gazizov R. K., Ibragimov N. H., Lukashchuk S. Yu. Nonlinear self-adjointness, conservation laws and exact solutions of time-fractional Kompaneets equations // Commun. Nonlin. Sci. Numer. Simulat., 2015. vol.23, no. 1-3. pp. 153-163. doi: 10.1016/j.cnsns.2014.11.010.

35. Лукащук С. Ю. О построении законов сохранения для интегро-дифференциальных уравнений дробного порядка // TMФ, 2015. Т. 184, №2. С. 179-199. doi: 10.4213/ tmf 8833.

36. Ибрагимов Н. Х., Авдонина Е. Д. Нелинейная самосопряженность, законы сохранения и построение решений уравнений в частных производных с помощью законов сохранения // УМH, 2013. Т. 68, №5(413). С. 111-146. doi : 10.4213/rm9536. 
MSC: 35R11, 76M60, 76S05

\title{
Group classification, invariant solutions and conservation laws of nonlinear orthotropic two-dimensional filtration equation with the Riemann-Liouville time-fractional derivative
}

\author{
V. O. Lukashchuk, S. Yu. Lukashchuk
}

Ufa State Aviation Technical University,

12, K. Marx st., Ufa, 450008, Russian Federation.

\begin{abstract}
A nonlinear two-dimensional orthotropic filtration equation with the Riemann-Liouville time-fractional derivative is considered. It is proved that this equation can admits only linear autonomous groups of point transformations. The Lie point symmetry group classification problem for the equation in question is solved with respect to coefficients of piezoconductivity. These coefficients are assumed to be functions of the square of the pressure gradient absolute value. It is proved that if the order of fractional differentiation is less than one then the considered equation with arbitrary coefficients admits a four-parameter group of point transformations in orthotropic case, and a five-parameter group in isotropic case. For the power-law piezoconductivity, the group admitted by the equation is five-parametric in orthotropic case, and six-parametric in isotropic case. Also, a special case of power function of piezoconductivity is determined for which there is an additional extension of admitted groups by the projective transformation. There is no an analogue of this case for the integer-order filtration equation. It is also shown that if the order of fractional differentiation $\alpha \in(1,2)$ then dimensions of admitted groups are incremented by one for all cases since an additional translation symmetry exists. This symmetry is corresponded to an additional particular solution of the fractional filtration equation under consideration.

Using the group classification results for orthotropic case, the representations of group-invariant solutions are obtained for two-dimensional subalgebras from optimal systems of symmetry subalgebras. Examples of reduced equations obtained by the symmetry reduction technique are given, and some exact solutions of these equations are presented.
\end{abstract}

\section{Research Article}

๑ ()(i) The content is published under the terms of the Creative Commons Attribution 4.0 International License (http://creativecommons.org/licenses/by/4.0/)

Please cite this article in press as:

Lukashchuk V. O., Luka shchuk S. Yu. Group classification, invariant solutions and conservation laws of nonlinear orthotropic two-dimensional filtration equation with the RiemannLiouville time-fractional derivative, Vestn. Samar. Gos. Tekhn. Univ., Ser. Fiz.-Mat. Nauki [J. Samara State Tech. Univ., Ser. Phys. Math. Sci.], 2020, vol. 24, no. 2, pp. 226-248. doi: 10.14498/vsgtu1761 (In Russian).

\section{Authors' Details:}

Veronika O. Lukashchuk (1) https://orcid.org/0000-0002-3082-1446

Cand. Phys. \& Math. Sci.; Associate Professor; Dept. of High Performance Computing Technologies and Systems; e-mail: voluks@gmail.com

Stanislav Yu. Lukashchuk (1) https://orcid.org/0000-0001-9209-5155

Dr. Phys. \& Math. Sci., Associate Professor; Professor; Dept. of High Performance Computing Technologies and Systems; e-mail: Isu@ugatu.su 
It is proved that the considered time-fractional filtration equation is nonlinearly self-adjoint and therefore the corresponding conservation laws can be constructed. The components of obtained conserved vectors are given in an explicit form.

Keywords: fractional filtration equation, group classification, Lie point symmetry, invariant solution, conservation law.

Received: $29^{\text {th }}$ November, 2019 / Revised: $17^{\text {th }}$ May, $2020 /$

Accepted: $1^{\text {st }}$ June, $2020 /$ First online: $30^{\text {th }}$ June, 2020

Competing interests. Authors declare no competing interests.

Authors' contributions and responsibilities. Each author has participated in article results and in the manuscript writing. The authors are absolutely responsible for submitting the final manuscript in print. Each author has approved the final version of manuscript.

Funding. This work was supported by the Ministry of Science and Higher Education of the Russian Federation (State task no. 1.3103.2017/4.6).

\section{References}

1. Samko S. G., Kilbas A. A., Marichev O. I. Fractional integrals and derivatives. Theory and applications. New York, Gordon \& Breach Sci. Publishers, 1993, xxxvi+976 pp.

2. Kilbas A. A., Srivastava H. M., Trujillo J. J. Theory and Applications of Fractional Differential Equations, North-Holland Mathematics Studies, vol. 204. Amsterdam, Elsevier, 2006, $\mathrm{xv}+523 \mathrm{pp}$.

3. Podlubny I. Fractional differential equations. An introduction to fractional derivatives, fractional differential equations, to methods of their solution and some of their applications, Mathematics in Science and Engineering, vol. 198. San Diego, Academic Press, 1999, xxiv +340 pp.

4. Metzler R., Klafter J. The random walk's guide to anomalous diffusion: a fractional dynamic approach, Phys. Rep., 2000, vol.339, no. 1, pp. 1-77. doi:10.1016/S0370-1573(00) 00070-3.

5. Hilfer R. Applications of fractional calculus in physics. Singapore, World Scientific, 2000, vii $+463 \mathrm{pp}$.

6. Nakhushev A. M. Drobnoe ischislenie $i$ ego primenenie [Fractional Calculus and its Applications]. Moscow, Fizmatlit, 2003, 272 pp. (In Russian)

7. Uchaikin V. V. Metod drobnykh proizvodnykh [Method of Fractional Derivatives]. Ul'yanovsk, Artishok, 2008, 512 pp. (In Russian)

8. Mainardi F. Fractional calculus and waves in linear viscoelasticity. An introduction to mathematical models. Hackensack, World Scientific, 2010, xx+347 pp.

9. Goloviznin V. M., Kondratenko P. S., Matveev L. V. Anomal'naia diffuziia radionuklidov $v$ sil'noneodnorodnykh geologicheskikh formatsiiakh [Anomalous Radionuclide Diffusion in Highly Heterogeneous Heological Formations]. Moscow, Nauka, 2010, 342 pp. (In Russian)

10. Fractional Dynamics: Recent Advances, eds. J. Klafter, S. C. Lim, R. Metzler. Hackensack, World Scientific, 2011, xiv+515 pp.

11. Fractional kinetics in solids: Anomalous charge transport in semiconductors, dielectrics and nanosystems, eds. V. Uchaikin, R. Sibatov. Boca Raton, CRC Press, 2013, xvi +257 pp. doi: $10.1142 / 8185$.

12. Baleanu D., Diethelm K., Scalas E., Trujillo J. J. Fractional calculus: models and numerical methods, Series on Complexity, Nonlinearity and Chaos, vol. 5. Hackensack, World Scientific, 2017, xxviii+448 pp. doi : 10.1142/10044. 
13. Ovsyannikov L. V. Group analysis of differential equations. New York, Academic Press, 1982, xvi+416 pp.

14. Olver P. J. Applications of Lie groups to differential equations, Graduate Texts in Mathematics, vol. 107. New York, Springer, 2000, xxviii+513 pp.

15. Bluman G. W., Cheviakov A. F., Anco S. C. Applications of symmetry methods to partial differential equations, Applied Mathematical Sciences, vol. 168. New York, Springer, 2010, xix +398 pp pp.. doi : 10.1007/978-0-387-68028-6.

16. Grigoriev Yu. N., Ibragimov N. H., Kovalev V. F., Meleshko S. V. Symmetries of integro-differential equations. With applications in mechanics and plasma physics., Lecture Notes in Physics, vol.806. Dordrecht, Springer, 2010, xiii +305 pp. doi: 10.1007/ 978-90-481-3797-8.

17. Ibragimov N. H. Transformation groups and Lie algebras. Hackensack, World Scientific, 2013, x+185 pp. doi: 10.1142/8763.

18. Gazizov R. K., Kasatkin A. A., Lukashchuk S. Y. Symmetries and group invariant solutions of fractional ordinary differential equations, In: Handbook of Fractional Calculus with Applications, Vol. 2 Fractional Differential Equations; eds. A. Kochubei, Y. Luchko. Berlin, De Gruyter, 2019, pp. 65-90. doi: 10.1515/9783110571660-004.

19. Gazizov R. K., Kasatkin A. A., Lukashchuk S. Y. Symmetries, conservation laws and group invariant solutions of fractional PDEs, In: Handbook of Fractional Calculus with Applications, Vol. 2 Fractional Differential Equations; eds. A. Kochubei, Y. Luchko. Berlin, De Gruyter, 2019, pp. 353-382. doi: 10.1515/9783110571660-016.

20. Raghavan R., Chen C. Fractional diffusion in rocks produced by horizontal wells with multiple, transverse hydraulic fractures of finite conductivity, J. Petrol. Sci. Eng., 2013, vol. 109, pp. 133-143. doi: 10.1016/j.petrol.2013.08.027.

21. Obembe A. D. Al-Yousef H. Y., Hossain M. E., Abu-Khamsin S. A. Fractional derivatives and their applications in reservoir engineering problems: A review, J. Petrol. Sci. Eng., 2017, vol. 157, pp. 312-327. doi: 10.1016/j.petrol.2017.07.035.

22. Gazizov R. K., Lukashchuk S. Yu. Fractional differentiation approach to modeling of fluid filtration processes in complex heterogeneous porous media, Vestnik UGATU, 2017, vol. 21, no. 4, pp. 104-112 (In Russian).

23. Babkov O. K. On group classification of some evolution equations, In: Book of abstracts of International conference MOGRAN-16 (October 28 - November 2, 2013). Ufa, Ufa State Aviation Technical Univ., 2013, pp. 6-7.

24. Ovsyannikov L. V. On the property of $x$-autonomy, Dokl. Math., 1993, vol. 47, no. 3, pp. 581584 .

25. Chirkunov Y. A. Linear autonomy conditions for the basic Lie algebra of a system of linear differential equations, Dokl. Math., 2009, vol.79, no.3, pp. 415-417. doi: 10.1134/ S1064562409030302.

26. Gazizov R. K., Kasatkin A. A., Lukashchuk S. Yu. Fractional differential equations: change of variables and nonlocal symmetries, Ufimsk. Mat. Zh., 2012, vol.4, no. 4, pp. 54-68 (In Russian).

27. Ibragimov N. H. A new conservation theorem, J. Math. Anal. Appl., 2007, vol.333, no.1, pp. 311-328. doi: 10.1016/j.jmaa.2006.10.078.

28. Ibragimov N. H. Nonlinear self-adjointness and conservation laws, J. Phys. A: Math. Theor., 2011, vol. 44, 432002. doi: 10.1088/1751-8113/44/43/432002.

29. Patera J., Winternitz P. Subalgebras of real three- and four-dimensional Lie algebras, J. Math. Phys., 1977, vol. 18, no.7, pp. 1449-1455. doi: 10.1063/1.523441.

30. Lukashchuk S. Yu. Symmetry reduction and invariant solutions for nonlinear fractional diffusion equation with a source term, Ufa Math. J., 2016, T. 8, № 4, C. 111-122. doi: 10 . 13108/2016-8-4-111.

31. Ovsyannikov L. V. On optimal systems of subalgebras, Dokl. Math., 1994, vol.48, no. 3, pp. 645-649. 
32. Chirkunov Yu. A., Khabirov S. V. Elementy simmetriinogo analiza differentsial'nykh uravnenii mekhaniki sploshnoi sredy [Elements of Symmetry Snalysis of Differential Equations of Continuum Mechanics]. Novosibirsk, Novosibirsk State Tecnical Univ., 2012, 659 pp. (In Russian)

33. Lukashchuk S. Yu. Conservation laws for time-fractional subdiffusion and diffusionwave equations, Nonlinear Dyn., 2015, vol.80, no.1-2, pp. 791-802. doi:10.1007/ s11071-015-1906-7.

34. Gazizov R. K., Ibragimov N. H., Lukashchuk S. Yu. Nonlinear self-adjointness, conservation laws and exact solutions of time-fractional Kompaneets equations, Commun. Nonlin. Sci. Numer. Simulat., 2015, vol. 23, no. 1-3, pp. 153-163. doi: 10.1016/j.cnsns. 2014.11.010.

35. Lukashchuk S. Y. Constructing conservation laws for fractional-order integro-differential equations, Theoret. and Math. Phys., 2015, vol.184, no.2, pp. 1049-1066. doi: 10.1007/ s11232-015-0317-8.

36. Ibragimov N. H., Avdonina E. D. Nonlinear self-adjointness, conservation laws, and the construction of solutions of partial differential equations using conservation laws, Russian Math. Surveys, 2013, vol. 68, no. 5, pp. 889-921. doi: 10.1070/RM2013v068n05ABEH004860. 